Revista Arbitrada Interdisciplinaria KOINONIA

Año VI. Vol VI. N${ }^{\circ}$. Edición Especial: Administración. 2021

Hecho el depósito de Ley: FA2016000010

ISSN: 2542-3088

FUNDACIÓN KOINONIA (F.K). Santa Ana de Coro. Venezuela.

Dayane Wendy Rejas-Phowell; Ana Cecilia Napán-Yactayo

http://dx.doi.org/10.35381/r.k.v6i1.1215

\title{
Clima organizacional y su relación con la satisfacción del cliente en la empresa ozonomedica
}

Organizational climate and its relationship with customer satisfaction in the ozonomedica company

\author{
Dayane Wendy Rejas-Phowell \\ 10008881@cientifica.edu.pe \\ Universidad Científica del Sur, Lima \\ Perú \\ https://orcid.org/0000-0001-9413-0815 \\ Ana Cecilia Napán-Yactayo \\ anapan@cientifica.edu.pe \\ Universidad Científica del Sur, Lima \\ Perú \\ https://orcid.org/0000-0002-5754-8355
}

Recepción: 20 de enero 2021

Revisado: 15 de febrero 2021

Aprobación: 20 de abril 2021

Publicación: 01 de mayo 2021 


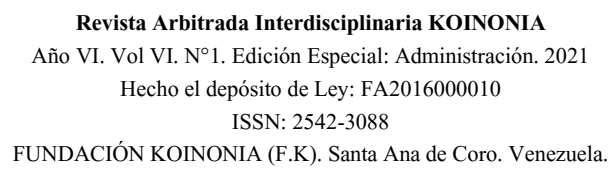

\title{
RESUMEN
}

El objetivo del estudio fue identificar la relación que existe entre el clima organizacional y la satisfacción del cliente en la empresa Ozonomedica en el distrito de la Molina del periodo 2021.La presente investigación fue de diseño no experimental, de tipo correlacional- descriptivo de corte transversal y de enfoque cuantitativo. Se tomó como población a los 100 trabajadores de la empresa de cara al público, la muestra estuvo conformada por 80 colaboradores de las diferentes áreas de servicio de la empresa. Los instrumentos obtuvieron un alfa de cronbach, CO (0.874) y SC (0.946). Los resultados indicaron una relación positiva alta moderada, con un Rho Spearman ( $\mathrm{r} s=0,735)$, siendo la prueba significativa con un pvalor $=0,000$ por debajo de la trazada en el estudio. Finalmente se corrobora la hipótesis general planteada, el cual muestra que existe una relación significativa entre las variables, donde a mayor clima organización, mayor será la satisfacción del cliente.

Descriptores: Cultura del trabajo; satisfacción en el trabajo; consumidor; motivación; condiciones de trabajo. (Palabras tomadas del Tesauro UNESCO).

\begin{abstract}
The objective of the study was to identify the influence that exists between the organizational climate and customer satisfaction in the Ozonomedica company in the district of La Molina in the period 2021. This research was of a non-experimental design, of a correlational-descriptive cross-sectional type and quantitative approach. The population was taken as the 100 workers of the company facing the public, the sample consisted of 80 collaborators from the different service areas of the company. The instruments obtained a cronbach's alpha, $\mathrm{CO}(0.87)$ and SC (0.94). The results indicated a moderate high positive relationship, with a Rho Spearman $r s=0.735$, the test being significant with a p-value $=0.000$ below that drawn in the study. Finally, the general hypothesis raised is corroborated, which shows that there is a significant relationship between the variables, where the greater the organizational climate, the greater the customer satisfaction.
\end{abstract}

Descriptors: Culture of work; job satisfaction; consumers; motivation; working conditions. (Words taken from UNESCO Thesaurus). 


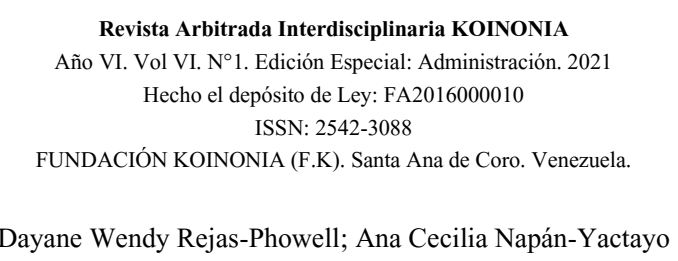

\section{INTRODUCCIÓN}

Desde hace ya varios años, la perspectiva con la cual se gestiona al personal ha venido cambiando a nivel mundial, ya no se piensa en trabajadores sino en colaboradores, en que todos los empleados de la empresa son parte de un engranaje, que apunta en una misma dirección como un equipo, esta nueva perspectiva se traduce en un interés de parte de la dirección de las empresas, en mantener al recurso humano contento y comprometido, para lo cual se debe tener un clima que sea agradable y permitiendo que los colaboradores desarrollen sus actividades de la mejor manera posible y con la mejor actitud; lógicamente las empresas que muestran un muy buen clima organizacional se han preocupado por generar las condiciones necesarias para que el trabajador puede ser lo más productivo posible.

De acuerdo con (Chiavenato, 2011) el clima organizacional son las cualidades y atributos, que tienen los colaboradores dentro de un ambiente laboral, las cuales son experimentadas y percibidas por los colaboradores que forman parte de la empresa, el cual influye significativamente en su conducta. Por otro lado, (Amaru, 2009) menciona que todos los componentes del clima organizacional de una empresa afectan las percepciones de los trabajadores, como por ejemplo la motivación, salarios, condiciones de trabajo, programa de incentivos, entre otros, todo ello puede o no afectar el rendimiento laboral, el cual podría generar sentimientos positivos, negativos 0 indiferencias dentro de la empresa.

En este sentido, (Litwin \& Stringer, 1968) consideraron el clima organizacional como una serie de factores ambientales relevantes como las creencias, actitudes, motivaciones y valores que el personal percibe dentro de la organización, los cuales influyen en su comportamiento laboral; además indica que estos pueden ser medidos en función a seis dimensiones como: estructura, responsabilidad individual, riesgo, sueldo, toma de decisión, tolerancia al conflicto y apoyo.

Por otra parte, (Robbins, 2009) menciona que el comportamiento de los trabajadores está determinado desde fuera, lo aprendido dentro de la empresa, además menciona que, 


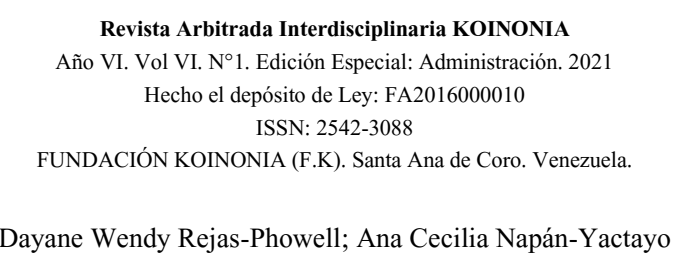

para tener una conducta deseada, debe haber un esfuerzo de parte de la organización, para premiar o castigar las conductas o trabajo realizado, teniendo así una probabilidad inferior de repetirse, en caso sea negativo.

En otras palabras, el clima organizacional seria la suma de características que los trabajadores perciben o experimentan dentro de su entorno laboral y que evidentemente influirá en su comportamiento dentro de la organización, por lo cual las empresas centran sus estrategias para mantener contentos y cómodos a sus trabajadores, teniendo en cuenta que de ello dependerá la rentabilidad de la empresa. Para ello se tomaron en cuenta las siguientes dimensiones: Condiciones Laborales, Motivación de los colaboradores y la Comunicación.

Para (Chiavenato, 2017) las condiciones laborales son las características físicas del puesto de trabajo como: instalaciones, distribución de los espacios de trabajo, salarios y las comodidades. Estas condiciones laborales pueden facilitar u obstaculizar su desempeño y la interacción entre grupos de trabajo. En complemento, (Amaru, 2009) menciona que la motivación en el trabajo es el resultado entre los motivos personales y los estímulos que genera su ambiente de trabajo, el cual puede influir positiva o negativamente en su desempeño laboral.

En contraposición, (Fernández, 2009) manifiesta que la comunicación es el conjunto de mensajes que se intercambian dentro de la organización el cual puede ser vertical $u$ horizontal, este debe mantenerse formal y centralizada para el logro de objetivos y su correcto cumplimiento de actividades de todo el personal. Además, (Kotler \& Keller, 2006), menciona que la satisfacción puede ser medida por tres dimensiones: nivel de satisfacción, rendimiento percibido y las expectativas.

En la investigación se ha tomado en cuenta tres dimensiones que evaluaran el grado de satisfacción del cliente: Atención al cliente, Valor percibido y Confianza. (Serna, 2006) refiere que un cliente se encuentra satisfecho después de recibir un servicio y atención por parte de los trabajadores de una empresa, este tiene que haber cumplido con sus expectativas, determinando las estrategias que las empresas elaboran para 


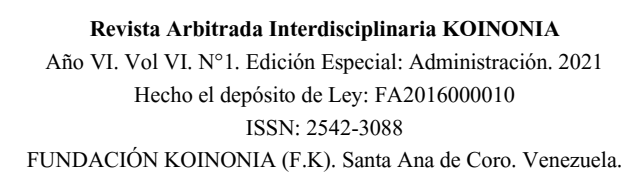

Dayane Wendy Rejas-Phowell; Ana Cecilia Napán-Yactayo

satisfacer al cliente, teniendo como resultado la preferencia y dejando de lado a la competencia. Para Zeithalml (1988) es un "valor percibido es la evaluación general del consumidor de la utilidad de un producto en función de las percepciones de lo que se recibe y lo que se da" (p.14).

El valor percibido es la evaluación que los clientes hacen de un producto o servicio, el cual muestra si satisface o no sus necesidades y expectativas. Asimismo, (Sutter \& Kocher, 2007) refieren que la confianza es toda buena voluntad de trasladar valores favorables hacia otras personas, esperando que sea devuelta o pueda ser respondida de forma similar o incluso mejor. La confianza es la fe o esperanza que se tiene respecto a otra persona, entidad o grupo. Esta voluntad es aceptada, luego de ya haber recibido algún producto o servicio, que comúnmente las organizaciones transmiten a sus clientes, este también puede ser transmitido por una tercera persona, el cual pudo crear ese lazo por medio de su experiencia.

Como antecedentes existen estudios realizados sobre el clima organizacional y la satisfacción del cliente, como la de (Castillo, 2019) comenta que la percepción de los funcionarios y la satisfacción que existe en el área de salud, sin embargo, se identifica que la percepción, es crítica y se recomienda mejorar aspectos referentes al ambiente físico de su centro de labores. En complemento, (Fernández-Oliva, et al. 2017) indica que, entre los resultados de su investigación, se destaca que el trato del personal médico, no contaba con una buena aceptación, mostrando así que la influencia de un clima laboral insatisfecho, genera por lo consiguiente una insatisfacción de los usuarios. En contraposición, (Baena-Arroyo, et al. 2016) destacan que el valor percibido se considera un elemento determinante para conseguir la satisfacción de los clientes en las actividades deportivas de los centros fitness.

Es por ello que muchas de las organizaciones se preocupan por generar mejores ambientes laborales, el cual tiene como resultado mejorar la productividad de sus colaboradores, donde finalmente se logrará la ansiada satisfacción de sus clientes, mitigando así la competencia; en ese sentido, se planteó el problema de investigación: 
¿De qué manera el clima organizacional se relaciona con la satisfacción del cliente en la empresa Ozonomedica -La Molina 2021?

Para responder a la interrogante planteamos la hipótesis: El Clima Organizacional se relaciona significativamente con la satisfacción del cliente en la empresa Ozonomedica La Molina, 2020. Para contrastar la hipótesis científica se ha planteado alcanzar el siguiente objetivo: Identificar si el clima organizacional se relaciona con la satisfacción del cliente en la empresa Ozonomedica - La Molina, 2021. El presente estudio se justifica de manera práctica, ya que ayudará a resolver el problema relacionado al clima organizacional y satisfacción del cliente que ha presentado la empresa Ozonomedica. Por lo que ayudará a encontrar soluciones concretas y se podrá tomar medidas estratégicas para corregir las situaciones que se mencionan como parte del problema, además de contribuir con alcances de valor y ser una base para futuros estudios que realice la empresa y estudios de terceros que se relacionen con cualquiera de las variables de investigación.

El estudio se da de acuerdo a las pautas del proceso de aplicación del método científico, lo que le brinda validez y confiablidad en sus resultados. El método se refleja en la forma en cómo se levanta información de acuerdo con los objetivos planteados, a través de la aplicación de un enfoque cuantitativo, plasmado en la utilización de un cuestionario del tipo estructurado de elaboración propia. Con el uso de esta información se procederá a validar la veracidad de las hipótesis y la relación entre las variables de estudio. Al medir la correlación entre las variables de estudio, se podrá apreciar la dimensión y la relación entre el clima organizacional sobre la satisfacción al cliente.

En virtud de lo planteado, la investigación tiene por objetivo identificar la relación que existe entre el clima organizacional y la satisfacción del cliente en la empresa Ozonomedica en el distrito de la Molina del periodo 2021. 


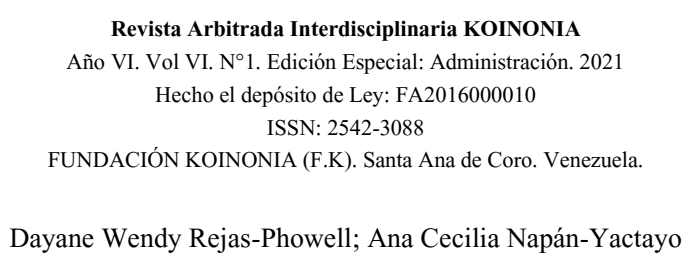

\section{MÉTODO}

La presente investigación fue de diseño no experimental de tipo correlacional- descriptivo de corte transversal de enfoque cuantitativo, de acuerdo con (Hernández, et al. 2014) la investigación no experimental se basa en estudios que se han realizado sin que exista manipulación deliberada de las variables y en los cuales sólo se observan los fenómenos en su ambiente natural.

La población según (Kotler \& Armstrong, 2012) es el estudio en términos de magnitud, los cuales son grupos considerados para un estudio estadístico, la cual para la investigación fueron 100 trabajadores conformado por cirujanos, personal encargado de estética, recepcionistas, cosmetólogas entre otros, que atienden directamente a clientes, ubicada en el distrito de La Molina.

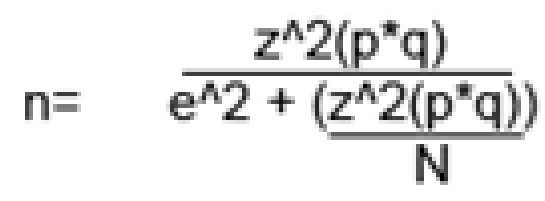

Dónde: $\mathrm{n}$ = Muestra (definida $\times$ la selección), $\mathrm{N}$ = Población finita (100) colaboradores $Z=$ Nivel de confianza $95 \%=(1.96) p=$ Probabilidad de acierto $(50 \%) q=$ Probabilidad de fracaso $(50 \%) E=$ Error $(5 \%)$

Por lo tanto, el tamaño de muestra por proporciones correspondió a 80 colaboradores de las áreas de cara al público, quienes fueron tomados al azar, el método de selección de la muestra fue muestreo probabilístico.

El cuestionario estuvo conformado por 18 ítems, para medir la variable clima organizacional se tomó como autor principal a Chiavenato (2011) de las cuales 9 corresponden a las siguientes dimensiones: las condiciones laborales, motivación de los colaboradores y la comunicación. Para medir la variable Satisfacción del cliente se tomó como autor principal a (Kotler \& Armstrong, 2012) de las cuales 09 corresponden a las siguientes dimensiones: atención al cliente, valor percibido y la confianza. 


$$
\begin{gathered}
\text { Revista Arbitrada Interdisciplinaria KOINONIA } \\
\text { Año VI. Vol VI. Nº1. Edición Especial: Administración. } 2021 \\
\text { Hecho el depósito de Ley: FA2016000010 } \\
\text { ISSN: 2542-3088 } \\
\text { FUNDACIÓN KOINONIA (F.K). Santa Ana de Coro. Venezuela. }
\end{gathered}
$$

Dayane Wendy Rejas-Phowell; Ana Cecilia Napán-Yactayo

En la tabla 1, se muestra la escala de valoración de los instrumentos, que está dado en una escala de Likert del 1 al 5, de la siguiente manera: Totalmente en desacuerdo (01); Desacuerdo (02); Indiferente (03); De acuerdo (04); Totalmente de acuerdo (05). El nivel de cada una de las variables será puntuado de acuerdo a las siguientes escalas:

Tabla 1. Baremos nivel y rango

\begin{tabular}{lc}
\hline Clima Organizacional & Satisfacción del Cliente \\
\hline & \\
$09-21>$ Nivel Bajo & $09-21>$ Nivel Bajo \\
$22-34>$ Nivel Medio & $22-34>$ Nivel Medio \\
$35-45>$ Nivel Alto & $35-45>$ Nivel Alto \\
\end{tabular}

La confiabilidad del instrumento fue a través del Alfa de Cronbach ( $\alpha$ ) y la validez por juicio de expertos. Se realizó una prueba piloto, además sirvió, para probar los tiempos en resolver las encuestas. En la tabla 2, el instrumento 1, Clima Organizacional encontramos los resultados respecto a la prueba de confiabilidad; el coeficiente de Alfa de Cronbach ( $\alpha$ ) es de 0,874 apreciando el grado de fiabilidad como adecuada. En el instrumento 2, Satisfacción del Cliente encontramos los resultados respecto a la prueba de confiabilidad; el coeficiente de Alfa de Cronbach ( $\alpha$ ) es de 0,946 considerando al instrumento de poseer una fiabilidad adecuada. 


$$
\begin{gathered}
\text { Revista Arbitrada Interdisciplinaria KOINONIA } \\
\text { Año VI. Vol VI. N} 1 \text {. Edición Especial: Administración. } 2021 \\
\text { Hecho el depósito de Ley: FA2016000010 } \\
\text { ISSN: } 2542-3088 \\
\text { FUNDACIÓN KOINONIA (F.K). Santa Ana de Coro. Venezuela. } \\
\text { Dayane Wendy Rejas-Phowell; Ana Cecilia Napán-Yactayo }
\end{gathered}
$$

Tabla 2. Confiabilidad del instrumento

\begin{tabular}{lc}
\hline \multicolumn{1}{c}{ Instrumento } & Alfa de Cronbach \\
\hline Clima organizacional & $(0.874)$ \\
Satisfacción del cliente & $(0.946)$ \\
\hline
\end{tabular}

En la tabla 2 para lo referente a la confiabilidad estadística, se va a utilizar el alfa de Cronbach para ver la estabilidad entre las alternativas que conforman la encuesta.

\section{RESULTADOS}

\section{a. Análisis descriptivo}

Tabla 1. Frecuencia de Rango de Edad

\begin{tabular}{ccc}
\hline Rango de edad & Frecuencia & Porcentaje \\
\hline $20-30$ & 41 & $51,3 \%$ \\
$31-40$ & 31 & $38,7 \%$ \\
$41-50$ & 8 & $10 \%$ \\
\hline Total & 80 & $100 \%$ \\
\hline
\end{tabular}

Fuente: Elaboración propia

En la tabla 3 referida a edad, se observa que la mayoría de los encuestados corresponde el rango de 20 a 30 años con un $51,2 \%$, seguido de 31 a 40 años con $38,7 \%$ y de 41 a 50 años con $10 \%$. 
Revista Arbitrada Interdisciplinaria KOINONIA

Año VI. Vol VI. N 1. Edición Especial: Administración. 2021

Hecho el depósito de Ley: FA2016000010

ISSN: 2542-3088

FUNDACIÓN KOINONIA (F.K). Santa Ana de Coro. Venezuela.

Dayane Wendy Rejas-Phowell; Ana Cecilia Napán-Yactayo

Tabla 2. Rango de Sexo

\begin{tabular}{ccc}
\hline $\begin{array}{c}\text { Rango de } \\
\text { Sexo }\end{array}$ & Frecuencia & Porcentaje \\
\hline F & 34 & $43 \%$ \\
M & 46 & $57 \%$ \\
\hline Total & 80 & $100 \%$ \\
\hline
\end{tabular}

Fuente: Elaboración propia

Según la tabla 4 referida a rango de sexo, se aprecia que 46 objetos de estudio corresponden al masculino con $57 \%$ de total de los encuestados, seguido de sexo femenino con $43 \%$.

Tabla 3. Baremo Nivel y Rango

\begin{tabular}{lc}
\hline Clima Organizacional & Satisfacción del Cliente \\
\hline $09-21->$ Nivel Bajo & $09-21->$ Nivel Bajo \\
$22-34->$ Nivel Medio & $22-34->$ Nivel Medio \\
$35-45->$ Nivel Alto & $35-45->$ Nivel Alto
\end{tabular}

Fuente: Elaboración propia 


$$
\begin{gathered}
\text { Revista Arbitrada Interdisciplinaria KOINONIA } \\
\text { Año VI. Vol VI. Nº } 1 \text {. Edición Especial: Administración. } 2021 \\
\text { Hecho el depósito de Ley: FA2016000010 } \\
\text { ISSN: 2542-3088 } \\
\text { FUNDACIÓN KOINONIA (F.K). Santa Ana de Coro. Venezuela. }
\end{gathered}
$$

Dayane Wendy Rejas-Phowell; Ana Cecilia Napán-Yactayo

En la tabla 5 se observa el baremo que nos da la valoración de los instrumentos según su nivel de respuestas.

Tabla 4. Frecuencia Clima Organizacional y su relación con la Satisfacción del Cliente

\begin{tabular}{lcccc}
\hline \multirow{2}{*}{ Escala } & \multicolumn{2}{c}{ Clima organizacional } & \multicolumn{2}{c}{ Satisfacción del cliente } \\
& Frecuencia & Porcentaje & Frecuencia & Porcentaje \\
\hline Bajo & 2 & $2.5 \%$ & 0 & $0 \%$ \\
Regular & 19 & $23.8 \%$ & 11 & $14 \%$ \\
Alto & 59 & $73.8 \%$ & 69 & $86 \%$ \\
Total & 80 & $100 \%$ & 80 & $100 \%$ \\
\hline
\end{tabular}

Fuente: Elaboración propia

En la tabla 6 se puede determinar que en relación a la variable Clima Organizacional el mayor porcentaje corresponde a la frecuencia con un alto nivel de 59 unidades objeto de estudio, el cual se logra obtener un $73.8 \%$ del total de los datos. Así mismo se observa que la variable Satisfacción del Cliente obtuvo un nivel alto con 69 unidades objeto de estudio, siendo así un $86 \%$ del total de la frecuencia estimada.

Tabla 5. Relación Las Condiciones Laborales y la Satisfacción del Cliente

\begin{tabular}{lcccc}
\hline & \multicolumn{2}{c}{ Las Condiciones Laborales } & \multicolumn{2}{c}{ Satisfacción Del Cliente } \\
\multicolumn{1}{c}{ Escala } & Frecuencia & Porcentaje & Frecuencia & Porcentaje \\
\hline Bajo & 3 & $3.8 \%$ & 0 & $0 \%$ \\
Regular & 17 & $21.3 \%$ & 11 & $14 \%$ \\
Alto & 60 & $75.0 \%$ & 69 & $86 \%$ \\
Total & 80 & $100.0 \%$ & 80 & $100 \%$ \\
\hline
\end{tabular}

Fuente: Elaboración propia 


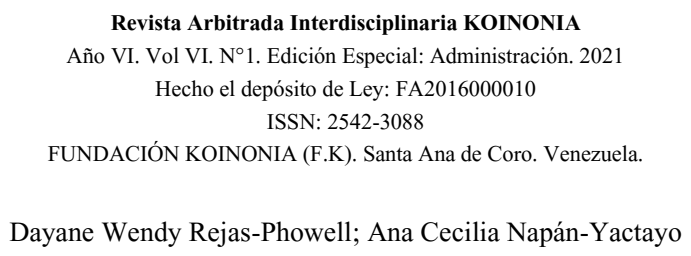

Dayane Wendy Rejas-Phowell; Ana Cecilia Napán-Yactayo

En la tabla 7 se observa la relación entre la dimensión Condiciones Laborales y la variable Satisfacción del Cliente, la cual especifica que hay una recurrencia en el nivel alto que representa el $75 \%$ del total de la frecuencia de la dimensión. Así mismo, se observa que en la variable de Satisfacción del Cliente el mayor porcentaje se presenta en la escala alta con un $86 \%$ del total de la frecuencia.

Tabla 6. Relación La motivación y la satisfacción del cliente

\begin{tabular}{lrrrr}
\hline & \multicolumn{2}{c}{$\begin{array}{c}\text { Motivación de los } \\
\text { Colaboradores }\end{array}$} & \multicolumn{2}{c}{ Satisfacción del cliente } \\
\cline { 2 - 5 } \multicolumn{1}{c}{ Escala } & Frecuencia & Porcentaje & Frecuencia & Porcentaje \\
\hline Bajo & 4 & $5.0 \%$ & 0 & $0 \%$ \\
Regular & 30 & $37.5 \%$ & 11 & $14 \%$ \\
Alto & 46 & $57.5 \%$ & 69 & $86 \%$ \\
Total & 80 & $100 \%$ & 80 & $100 \%$ \\
\hline
\end{tabular}

Fuente: Elaboración propia

Según la tabla 8 se aprecia que la relación entre la dimensión Motivación de los Colaboradores y la variable Satisfacción del cliente, tiene una recurrencia en el nivel alto que representa el $58 \%$ del total de frecuencia de la dimensión. En cuanto a la variable Satisfacción del Cliente el mayor porcentaje se presenta en la escala alta con un $86 \%$ del total de la frecuencia. 
Revista Arbitrada Interdisciplinaria KOINONIA

Año VI. Vol VI. N 1 . Edición Especial: Administración. 2021

Hecho el depósito de Ley: FA2016000010

ISSN: 2542-3088

FUNDACIÓN KOINONIA (F.K). Santa Ana de Coro. Venezuela.

Dayane Wendy Rejas-Phowell; Ana Cecilia Napán-Yactayo

Tabla 7. Relación La comunicación y satisfacción del cliente

\begin{tabular}{lrrrr}
\hline & \multicolumn{2}{c}{ La Comunicación } & \multicolumn{2}{c}{ Satisfacción del Cliente } \\
\cline { 2 - 5 } Escala & Frecuencia & Porcentaje & Frecuencia & Porcentaje \\
\hline Bajo & 3 & $4 \%$ & 0 & $0 \%$ \\
Regular & 17 & $21 \%$ & 11 & $14 \%$ \\
Alto & 60 & $75 \%$ & 69 & $86 \%$ \\
Total & 80 & $100 \%$ & 80 & $100 \%$ \\
\hline
\end{tabular}

Fuente: Elaboración propia

En la tabla 9, se observa que la relación entre la comunicación y la Satisfacción del cliente cuenta con una recurrencia en el nivel alto de $17 \%$ del total de la dimensión. Así mismo se aprecia que la variable de Satisfacción del Cliente se mantiene en el nivel alto con una frecuencia de $86 \%$ del total de objeto de estudio.

Tabla 8. Dimensiones de la Variable Clima Organizacional

\begin{tabular}{|c|c|c|c|c|c|c|c|}
\hline \multirow{2}{*}{ Dimensión } & \multicolumn{3}{|c|}{ Bajo } & \multicolumn{2}{|c|}{ Regular } & Alto & \multirow[b]{2}{*}{$\begin{array}{l}\text { Total, de } \\
\text { frecuencia }\end{array}$} \\
\hline & $\mathrm{Fr}$ & $\%$ & $\mathrm{Fr}$ & $\%$ & $\mathrm{Fr}$ & $\%$ & \\
\hline $\begin{array}{l}\text { Condiciones } \\
\text { Laborales } \\
\text { Motivación de }\end{array}$ & 3 & $4 \%$ & 17 & $21 \%$ & 60 & $75 \%$ & 80 \\
\hline $\begin{array}{l}\text { los } \\
\text { Colaboradores }\end{array}$ & 4 & $5 \%$ & 30 & $38 \%$ & 46 & $58 \%$ & 80 \\
\hline Comunicación & 3 & $4 \%$ & 17 & $21 \%$ & 60 & $75 \%$ & 80 \\
\hline
\end{tabular}

Fuente: Elaboración propia 


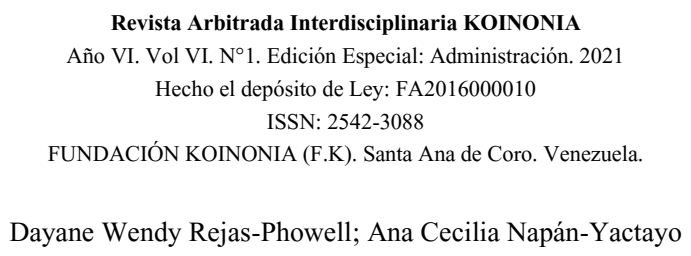

Dayane Wendy Rejas-Phowell; Ana Cecilia Napán-Yactayo

Según la tabla 10 se aprecia la frecuencia entre las dimensiones de la variable de Clima Organizacional:

Con respecto a las condiciones Laborales, se observa que en el nivel alto tiene un porcentaje de aceptación de $75 \%$, seguido de la comunicación con $75 \%$ y finalmente la motivación de los colaboradores en un porcentaje moderado de $58 \%$.

Tabla 9. Dimensiones de la variable satisfacción del cliente

\begin{tabular}{|c|c|c|c|c|c|c|c|}
\hline \multirow{2}{*}{ Dimensión } & \multicolumn{2}{|c|}{ Bajo } & \multicolumn{2}{|c|}{ Regular } & \multicolumn{2}{|c|}{ Alto } & Total, de \\
\hline & Frecuencia & Porcentaje & Frecuencia & Porcentaje & Frecuencia & Porcentaje & frecuencia \\
\hline $\begin{array}{l}\text { Atención } \\
\text { del Cliente }\end{array}$ & 3 & $4 \%$ & 16 & $20 \%$ & 61 & $76 \%$ & 80 \\
\hline $\begin{array}{l}\text { Valor } \\
\text { percibido }\end{array}$ & 0 & $0 \%$ & 12 & $15 \%$ & 68 & $85 \%$ & 80 \\
\hline Confianza & 1 & $1 \%$ & 15 & $19 \%$ & 64 & $80 \%$ & 80 \\
\hline
\end{tabular}

Fuente: Elaboración propia

Según la tabla 11, se observa los niveles de frecuencia de las dimensiones de la Satisfacción del Cliente:

El Valor Percibido cuenta con un porcentaje de frecuencia de $85 \%$, seguido de la Confianza con $80 \%$ y finalmente Atención al Cliente con $76 \%$, el cual indica que todas ellas cuentan con aceptaciones moderadas altas con respecto a la Satisfacción del Cliente. 


$$
\begin{gathered}
\text { Revista Arbitrada Interdisciplinaria KOINONIA } \\
\text { Año VI. Vol VI. Nº1. Edición Especial: Administración. } 2021 \\
\text { Hecho el depósito de Ley: FA2016000010 } \\
\text { ISSN: 2542-3088 } \\
\text { FUNDACIÓN KOINONIA (F.K). Santa Ana de Coro. Venezuela. }
\end{gathered}
$$

Dayane Wendy Rejas-Phowell; Ana Cecilia Napán-Yactayo

\section{b. Análisis inferencial}

En el siguiente análisis se realizará la prueba de normalidad para establecer el estadístico que se emplear para comprobar nuestra hipótesis. Debido a que se tiene una muestra mayor a 50 elementos, utilizaremos el estadístico de Kolmogorov-Smirnov, el cual nos indicara si la distribución es normal o no, y si la prueba es para paramétrica o no.

En la tabla 12 se aprecia la prueba de normalidad con el estadístico de KolmogorovSmirnov, el cual se observa que ninguno de los elementos evaluados presenta distribución normal teniendo un $p=$ valor por debajo de la trazada en el estudio. Por lo tanto, se utilizará una prueba no paramétrico Rho de Spearman.

Tabla 12. Prueba de normalidad Kolmogorov-Smirnov

\begin{tabular}{lccc}
\hline \multirow{2}{*}{ Variable / dimensión } & \multicolumn{3}{c}{ Kolmogorov-Smirnov $^{\mathrm{a}}$} \\
\cline { 2 - 4 } Condiciones Laborales & Estadístico & gl & Sig. \\
Motivación de los Colaboradores &, 141 & 80 &, 000 \\
Comunicación &, 173 & 80 &, 000 \\
Clima Organizacional &, 164 & 80 &, 000 \\
Satisfacción del Cliente &, 146 & 80 &, 000 \\
\hline a. Corrección de significación de Lilliefors & & &, 000
\end{tabular}

Fuente: Elaboración propia

\section{c. Prueba de correlación}

Continuando con el proceso de la investigación se efectúa la prueba de correlación de Kolmogorov-Smirnova debido a que las variables son cuantitativas y para fines de operacionalización han sido trabajadas en escala de Likert para frecuencia y puntajes. No se puede asumir la existencia de distribución normal en el conjunto de los datos obtenido. 


$$
\begin{gathered}
\text { Revista Arbitrada Interdisciplinaria KOINONIA } \\
\text { Año VI. Vol VI. N} 1 \text {. Edición Especial: Administración. } 2021 \\
\text { Hecho el depósito de Ley: FA2016000010 } \\
\text { ISSN: } 2542-3088 \\
\text { FUNDACIÓN KOINONIA (F.K). Santa Ana de Coro. Venezuela. } \\
\text { Dayane Wendy Rejas-Phowell; Ana Cecilia Napán-Yactayo }
\end{gathered}
$$

\section{Objetivo general:}

Identificar si el clima organizacional se relaciona con la satisfacción del cliente en la empresa Ozonomedica - La Molina, 2021.

\section{Hipótesis general:}

El Clima Organizacional se relación significativamente con la satisfacción del cliente en la empresa Ozonomedica - La Molina, 2021.

Como se muestra en la tabla 13, la relación entre las variables Clima Organizacional y Satisfacción del cliente es estadísticamente significativa, debido a que se obtuvo un $p$ valor $=0,000$ y siendo $p<0,050$. El coeficiente Rho de Spearman, arroja un $r s=0,735$, lo cual refleja una relación positiva y fuerte de manera bilateral, es decir, mientas mayor sea

\begin{tabular}{|c|c|c|c|c|}
\hline & & & $\begin{array}{c}\text { Clima } \\
\text { Organizacional }\end{array}$ & $\begin{array}{l}\text { Satisfacción } \\
\text { del Cliente }\end{array}$ \\
\hline \multirow[t]{6}{*}{$\begin{array}{l}\text { Rho de } \\
\text { Spearman }\end{array}$} & \multirow{3}{*}{$\begin{array}{c}\text { Clima } \\
\text { Organizacional }\end{array}$} & $\begin{array}{l}\text { Coeficiente de } \\
\text { correlación }\end{array}$ & 1,000 &, $735^{\circ-}$ \\
\hline & & Sig. (bilateral) & - &, 000 \\
\hline & & $\mathrm{N}$ & 80 & 80 \\
\hline & \multirow{3}{*}{$\begin{array}{c}\text { Satisfacción del } \\
\text { Cliente }\end{array}$} & $\begin{array}{l}\text { Coeficiente de } \\
\text { correlación }\end{array}$ &, $735^{*}$ & 1,000 \\
\hline & & Sig. (bilateral) &, 000 & - \\
\hline & & $\mathrm{N}$ & 80 & 80 \\
\hline
\end{tabular}
el Clima Organizacional, mayor será la Satisfacción del Cliente. Se acepta la hipótesis alterna "El Clima Organizacional se relaciona significativamente con la satisfacción del cliente en la empresa Ozonomedica - La Molina, 2021".

Tabla 13. Correlación clima organizacional y satisfacción del cliente ]

**. La correlación es significativa en el nivel 0,01 (bilateral).

Fuente: Elaboración propia 


$$
\begin{gathered}
\text { Revista Arbitrada Interdisciplinaria KOINONIA } \\
\text { Año VI. Vol VI. Nº1. Edición Especial: Administración. } 2021 \\
\text { Hecho el depósito de Ley: FA2016000010 } \\
\text { ISSN: 2542-3088 } \\
\text { FUNDACIÓN KOINONIA (F.K). Santa Ana de Coro. Venezuela. }
\end{gathered}
$$

Dayane Wendy Rejas-Phowell; Ana Cecilia Napán-Yactayo

\section{Objetivo específico 1}

Determinar si las condiciones laborales se relacionan con la satisfacción del cliente en la empresa Ozonomedica la Molina, 2021.

\section{Hipótesis específica 1}

Las condiciones laborales se relacionan significativamente con la satisfacción del cliente en la empresa Ozonomedica - La Molina, 2020.

Según la tabla 14 La hipótesis alterna plantea que Condiciones Laborales se relacionan significativamente con la satisfacción del cliente en la empresa Ozonomedica - La Molina,

\begin{tabular}{|c|c|c|c|c|}
\hline \multicolumn{5}{|c|}{ Correlaciones } \\
\hline & & & $\begin{array}{c}\text { Condiciones } \\
\text { Laborales }\end{array}$ & $\begin{array}{l}\text { Satisfacción } \\
\text { del Cliente }\end{array}$ \\
\hline \multirow[t]{6}{*}{$\begin{array}{l}\text { Rho de } \\
\text { Spearman }\end{array}$} & & $\begin{array}{l}\text { Coeficiente de } \\
\text { correlación }\end{array}$ & 1,000 & $670^{*}$ \\
\hline & Condiciones Laborales & Sig. (bilateral) & - &, 000 \\
\hline & & $\mathrm{N}$ & 80 & 80 \\
\hline & & $\begin{array}{l}\text { Coeficiente de } \\
\text { correlación }\end{array}$ & $670^{*}$ & 1,000 \\
\hline & Satisfacción del Cliente & Sig. (bilateral) &, 000 & - \\
\hline & & $\mathrm{N}$ & 80 & 80 \\
\hline
\end{tabular}
2021, en los resultados estadísticos se puede apreciar que el coeficiente de correlación arroja un valor considerado moderado ( $\mathrm{rs}=0,670$ ), por lo tanto se acepta la hipótesis especifica 1.

Tabla 14. Correlación condiciones laborales y satisfacción del cliente

**. La correlación es significativa en el nivel 0,01 (bilateral).

Fuente: Elaboración propia 


$$
\begin{gathered}
\text { Revista Arbitrada Interdisciplinaria KOINONIA } \\
\text { Año VI. Vol VI. Nº1. Edición Especial: Administración. } 2021 \\
\text { Hecho el depósito de Ley: FA2016000010 } \\
\text { ISSN: 2542-3088 } \\
\text { FUNDACIÓN KOINONIA (F.K). Santa Ana de Coro. Venezuela. }
\end{gathered}
$$

Dayane Wendy Rejas-Phowell; Ana Cecilia Napán-Yactayo

\section{Objetivo específico 2}

Determinar si la motivación de los colaboradores se relaciona con la satisfacción del cliente en la empresa Ozonomedica La Molina, 2021.

\section{Hipótesis específica 2}

La motivación de los colaboradores se relaciona significativamente con la satisfacción del cliente en la empresa Ozonomedica -La Molina, 2021.

En la tabla 15 de correlación entre Motivación de los Colaboradores y Satisfacción del Cliente se aprecia que el coeficiente de Rho Spearman obtuvo un valor de "rs $=0,680$ " lo cual indica una correlación moderada entre la dimensión y la variable. Donde sí la dimensión Motivación de los Colaboradores incrementa su valor, también lo hará la

\begin{tabular}{|c|c|c|c|c|}
\hline & & & $\begin{array}{c}\text { Motivación de los } \\
\text { Colaboradores }\end{array}$ & $\begin{array}{l}\text { Satisfacción } \\
\text { del Cliente }\end{array}$ \\
\hline \multirow[t]{6}{*}{$\begin{array}{l}\text { Rho de } \\
\text { Spearman }\end{array}$} & \multirow{3}{*}{$\begin{array}{c}\text { Motivación de los } \\
\text { Colaboradores }\end{array}$} & $\begin{array}{l}\text { Coeficiente de } \\
\text { correlación }\end{array}$ & 1,000 &, $680^{-*}$ \\
\hline & & Sig. (bilateral) & . &, 000 \\
\hline & & $\mathrm{N}$ & 80 & 80 \\
\hline & \multirow{3}{*}{$\begin{array}{l}\text { Satisfacción del } \\
\text { Cliente }\end{array}$} & $\begin{array}{l}\text { Coeficiente de } \\
\text { correlación }\end{array}$ & $680^{*}$ & 1,000 \\
\hline & & Sig. (bilateral) &, 000 & - \\
\hline & & $\mathrm{N}$ & 80 & 80 \\
\hline
\end{tabular}
variable Satisfacción del Cliente. Se acepta la hipótesis alterna "La motivación de los colaboradores se relaciona significativamente con la satisfacción del cliente en la empresa Ozonomedica -La Molina, 2021".

Tabla 15. Correlación motivación de los colaboradores y satisfacción del cliente

***. La correlación es significativa en el nivel 0,01 (bilateral).

Fuente: Elaboración propia 


$$
\begin{gathered}
\text { Revista Arbitrada Interdisciplinaria KOINONIA } \\
\text { Año VI. Vol VI. Nº1. Edición Especial: Administración. } 2021 \\
\text { Hecho el depósito de Ley: FA2016000010 } \\
\text { ISSN: 2542-3088 } \\
\text { FUNDACIÓN KOINONIA (F.K). Santa Ana de Coro. Venezuela. }
\end{gathered}
$$

Dayane Wendy Rejas-Phowell; Ana Cecilia Napán-Yactayo

\section{Objetivo específico 3}

Determinar si la comunicación se relaciona con la satisfacción del cliente en la empresa Ozonomedica La Molina, 2021.

\section{Hipótesis específica 3}

La comunicación se relaciona significativamente con la satisfacción del cliente en la empresa Ozonomedica -La Molina, 2021

Según la tabla 16 La hipótesis alterna plantea que la Comunicación se relaciona significativamente con la satisfacción del cliente en la empresa Ozonomedica - La

\begin{tabular}{|c|c|c|c|c|}
\hline \multicolumn{5}{|c|}{ Correlaciones } \\
\hline & & & Comunicación & $\begin{array}{l}\text { Satisfacción } \\
\text { del Cliente }\end{array}$ \\
\hline \multirow[t]{6}{*}{$\begin{array}{l}\text { Rho de } \\
\text { Spearman }\end{array}$} & \multirow{3}{*}{ Comunicación } & $\begin{array}{l}\text { Coeficiente de } \\
\text { correlación }\end{array}$ & 1,000 &, $569^{*}$ \\
\hline & & Sig. (bilateral) & - &, 000 \\
\hline & & $\mathrm{N}$ & 80 & 80 \\
\hline & \multirow{3}{*}{$\begin{array}{l}\text { Satisfacción del } \\
\text { Cliente }\end{array}$} & $\begin{array}{l}\text { Coeficiente de } \\
\text { correlación }\end{array}$ &, $569^{*}$ & 1,000 \\
\hline & & Sig. (bilateral) &, 000 & \\
\hline & & $N$ & 80 & 80 \\
\hline
\end{tabular}
Molina, 2021, en los resultados estadísticos se puede apreciar que el coeficiente de correlación arroja un valor considerado moderado $(r s=0,569)$, por lo tanto, se acepta la hipótesis especifica 3.

Tabla 13. Correlación comunicación y satisfacción del cliente

**. La correlación es significativa en el nivel 0,01 (bilateral).

Fuente: Elaboración propia 


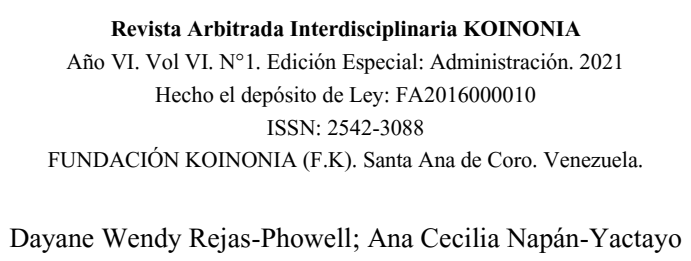

\section{DISCUSIÓN}

En el presente trabajo se planteó determinar la relación entre variables, de lo cual se formuló la siguiente hipótesis general: El Clima Organizacional se relaciona significativa con la satisfacción del cliente en la empresa Ozonomedica - La Molina, 2021. Como nos da a conocer el estudio de (Montoya-Cáceres, et al. 2017) sobre la importancia de tener un buen clima laboral dentro de las organizaciones, así mismo, se concuerda con (Castillo, 2019) quien en su investigación determinó la influencia que genera la constante preocupación sobre tener un alto clima organizacional

Por otro lado, (Guerrero-Bejarano, et al. 2019) mencionan que a mejor clima mejor será la satisfacción, no solo de la organización, sino también de los clientes, en complemento (Fernández-Oliva, et al. 2019) destacan que la remuneración tiene que ir acorde al esfuerzo requerido por cada colaborador, mientras que (Guerrero-Bejarano, et al. 2018) mencionan que las condiciones físicas que perciben los empleados, dan como resultado su satisfacción.

Siguiendo con lo planteado, (Castillo-Saavedra, et al. 2019) mencionan la importancia del incentivo y motivación que se le debe brindar la personal, obteniendo así un índice mayor de estabilidad laboral y la baja rotación del personal.

\section{CONCLUSIONES}

Se identificó que el clima organizacional si se relaciona con la satisfacción del cliente en la empresa Ozonomedica - La Molina, 2021, siento una correlación positiva moderada alta de 0,735 . Debido a que el buen ambiente representa una satisfacción no solo de manera interna hacia los colaboradores, sino también en la atención, el cual se ve reflejado en la aceptación y la fidelización de sus clientes, dejando de lado a la competencia.

Las condiciones laborales se relacionan con la satisfacción del cliente en la empresa Ozonomedica la Molina, 2021.Se logró evidenciar un grado de correlación positiva 


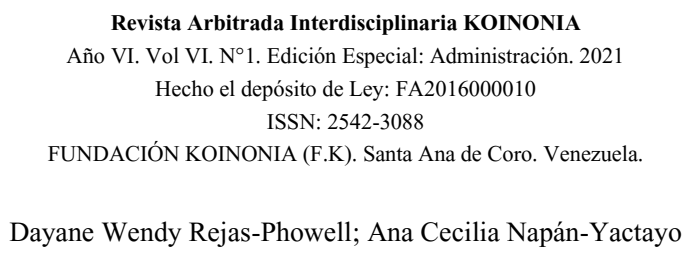

Dayane Wendy Rejas-Phowell; Ana Cecilia Napán-Yactayo

modera de 0,670 . Debido a que el $75 \%$ de los encuestados infieren que las condiciones en las que laboran casi siempre son satisfactorias y cumplen con lo esperado La motivación de los colaboradores si se relaciona con la satisfacción del cliente en la empresa Ozonomedica La Molina, 2021. Siendo representada por una correlación positiva modera de 0,680 . Debido a que el $57.5 \%$ de los encuestados, indicaron que casi siempre se sienten motivados con respecto a la organización y sus funciones realizadas dentro de su centro de labores.

La comunicación si se relaciona con la satisfacción del cliente en la empresa Ozonomedica La Molina, 2021. Logrando demostrar una correlación modera de 0,569. Debido a que los colaboradores indican que casi siempre la comunicación dentro de la organización se difunde, esto genera que se tenga conocimiento de las actividades que se les realiza a los clientes que recurren al centro estético a realizarse servicios de belleza.

\section{FINANCIAMIENTO}

No monetario

\section{AGRADECIMIENTOS}

A la Empresa Ozonomedica que brindo toda la información para el desarrollo de la investigación.

\section{REFERENCIAS CONSULTADAS}

Amaru, A. (2009). Fundamentos de administración [Management fundamentals] Pearson Educacion.

Baena-Arroyo, J, García-Fernández, J, Bernal-García, A, Lara-Bocanegra, A, \& GálvezRuíz, P. (2016). El valor percibido y la satisfacción del cliente en actividades dirigidas virtuales y con técnico en centros de fitness [Perceived value and customer satisfaction in virtual directed activities and with technicians in fitness centers]. Revista de Psicología del Deporte, 25(2),219-227. 


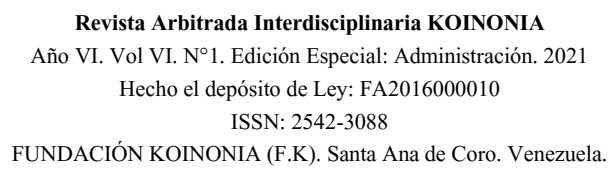

Castillo, M. (2019). Evaluación de la satisfacción al cliente interno y el clima organizacional en la unidad gestión de servicios generales del área de salud Paraíso Cervantes en el año 2019 [Evaluation of internal customer satisfaction and organizational climate in the general services management unit of the Paraíso Cervantes health area in 2019]. Trabajo de titulación de maestría. Recuperado de https://n9.cl/vchkz

Castillo-Saavedra, E, Medina-Reyes, Marleny-Alida, Bernardo-Trujillo, J, Reyes-Alfaro, C, \& Ayala-Jara, C. (2019). Liderazgo y clima organizacional en trabajadores de establecimientos de salud de una microred de Perú. [Leadership and organizational climate in workers of health establishments of a microgrid in Peru]. Revista Cubana de Salud Pública, 45(2), e1351.

Chiavenato, I. (2009). Comportamiento organizacional [Organizational behavior]. Segunda edición. McGRAW-HILL/ÍNTER AMERICAN A EDITORES, S.A. DE C.V.

Chiavenato, I. (2017). Introducción a la teoría general de la administración. [Introduction to the general theory of administration]. Décima edición. McGRAW-HILL/ÍNTER AMERICAN A EDITORES, S.A. DE C.V.

Fernández, C. (2009). La comunicación en las organizaciones. [Communication in organizations]. Trillas.

Fernández-Oliva, D., Revilla-Velásquez, M., Kolevic-Roca, L., Cabrejos-Castilla, I., Muchaypiña-Gallegos, I., Sayas-Avilés, I., Chávez-Conde, L., \& Mamani-Urrutia, V. (2019). Clima organizacional y satisfacción del usuario externo en los servicios de hospitalización del Instituto Nacional de Salud del Niño, 2017 [Organizational climate and external user satisfaction in the hospitalization services of the National Institute of Child Health, 2017]. Anales De La Facultad De Medicina, 80(2), 18892. https://doi.org/10.15381/anales.802.15745

Guerrero-Bejarano, M. A., Parra-Suárez, R. J., \& Arce-Vera, M. F. (2018). La satisfacción laboral y su efecto en la satisfacción del cliente, un análisis teórico [Job satisfaction and its effect on customer satisfaction, a theoretical analysis]. INNOVA Research Journal, 3(8), 140-146. https://doi.org/10.33890/innova.v3.n8.2018.879

Hernández, R., Fernández, C. y Batista, P. (2014). Metodologia de la investigacion. [Investigation methodology]. Sexta edición. McGRAW-HILL.

Kotler, P. \& Keller, K. (2012). Dirección de marketing. [Marketing direction]. Pearson Educación. 


$$
\begin{gathered}
\text { Revista Arbitrada Interdisciplinaria KOINONIA } \\
\text { Año VI. Vol VI. N}{ }^{\circ} \text {. Edición Especial: Administración. } 2021 \\
\text { Hecho el depósito de Ley: FA2016000010 } \\
\text { ISSN: 2542-3088 } \\
\text { FUNDACIÓN KOINONIA (F.K). Santa Ana de Coro. Venezuela. } \\
\text { Dayane Wendy Rejas-Phowell; Ana Cecilia Napán-Yactayo }
\end{gathered}
$$

Litwin, G. \& Stringer, R. (1968). Motivación y clima organizacional. [Motivation and organizational climate]. Graduate School of Bussines Administration, Harvard University.

Martínez, C. (2016). Estadística. [Statistics]. ECOE Ediciones

Montoya-Cáceres, P, Beiio-Escamlila, N, Bermúdez-Jara, N, Burgos-Ríos, F, FuentealbaSandoval, M, \& Padilla-Pérez, A. (2017). Satisfacción Laboral y su Relación con el Clima Organizacional en Funcionarios de una Universidad. [Job Satisfaction and its Relationship with the Organizational Climate in Officials of a University]. Ciencia\&trabajo, $19(58), 7-13 . \quad$ https://dx.doi.org/10.4067/S0718$\underline{24492017000100007}$

Robbins, S. (2009). Comportamiento organizacional. [ Organizational behavior]. Pearson Educación.

Serna, H. (2006). Conceptos básicos. En Servicio al cliente. [ Basic concepts. In Customer Service]. Panamericana editorial Ltda

Sutter, M., \& Kocher, M. G. (2007). Trust and trustworthiness across different age groups. Games and Economic Behavior, 59(2), 364382. https://doi.org/10.1016/..geb.2006.07.006

Zeithaml, V. (1988). Consumer Perceptions of Price, Quality, and Value: A Means-End Model and Synthesis of Evidence. Journal of Marketing, 52(3), 2-22. https://doi.org/10.2307/1251446 